\title{
A136 PLASMACYTOID DENDRITIC CELLS REGULATE THE MAGNITUDE OF AN AUTOIMMUNE RESPONSE THROUGH EXPANSION OF SUPPRESSIVE MONOCYTES
}

M loannou, ${ }^{1,2}$ T Alissafi, ${ }^{1,2}$ L Boon, ${ }^{3}$ S Deraos, ${ }^{4} \mathrm{~J}$ Matsoukas, ${ }^{4}$ D Boumpas, ${ }^{1,2}$ P Verginis ${ }^{1,2}{ }^{1}$ University of Crete, Medical School, Crete, Greece; ${ }^{2}$ Institute of Molecular Biology and Biotechnology, Foundation for Research and Technology, 71300; ${ }^{3}$ Bioceros, BV 3584 CM Utrecht, The Netherlands; ${ }^{4}$ Department of Chemistry, University of Patras, 26504, Patras, Greece

\subsection{6/ard.2010.129643m}

Myeloid-derived suppressor cells (MDSCs) are essential for the suppression of multiple immune responses. However, the mechanisms that regulate their expansion and suppressive function remain largely unknown. We demonstrate here that depletion of plasmacytoid dendritic cells (pDCs) resulted in the expansion of CD11b+Gr1+CD11c-CD19- myeloid cells in the peripheral lymphoid organs and suppression of autoimmune disease. pDC-depleted/ myelin oligodendrocyte glycoprotein (MOG)-immunised mice had reduced cellularity in the draining lymph nodes (DLNs) which was associated with decreased frequency of IAb-MOG+CD4+ effector $\mathrm{T}$ cells. In addition, DLN MOG-specific T cells from $\mathrm{pDC}$ depleted/MOG-immunised mice showed impaired proliferation and decreased interferon (IFN) $\gamma /$ interleukin (IL) 17 secretion in recall in vitro assays. Notably, pDC depletion resulted in recruitment of large numbers of MDSCs in the DLNs as well as the spleen of immunised mice, indicating their involvement in the suppression of EAE. In conclusion, our data demonstrate a crucial role of $\mathrm{pDC}$ in the priming of an autoimmune response which is associated with expansion and recruitment of suppressor monocytes in the peripheral lymphoid organs. Further characterisation of the molecular mechanism of such phenomenon will provide new insights into the immunoregulation of autoimmune and inflammatory diseases. 